\title{
Laser-intensity dependence of photoassociation in ultracold metastable helium
}

\author{
Daniel G. Cocks and Ian B. Whittingham \\ School of Mathematics, Physics and Information Technology, James Cook University, Townsville, Queensland 4811, Australia
}

(Received 30 April 2009; published 24 August 2009)

\begin{abstract}
Photoassociation of spin-polarized metastable helium to the three lowest rovibrational levels of the $J=1,0_{u}^{+}$ state asymptoting to $2 s{ }^{3} S_{1}+2 p{ }^{3} P_{0}$ is studied using a second-order perturbative treatment of the line shifts valid for low laser intensities, and two variants of a nonperturbative close-coupled treatment, one based upon dressed states of the matter plus laser system, and the other on a modified radiative coupling which vanishes asymptotically, thus simulating experimental conditions. These nonperturbative treatments are valid for arbitrary laser intensities and yield the complete photoassociation resonance profile. Both variants give nearly identical results for the line shifts and widths of the resonances and show that their dependence upon laser intensity is very close to linear and quadratic, respectively, for the two lowest levels. The resonance profiles are superimposed upon a significant background loss, a feature for this metastable helium system not present in studies of photoassociation in other systems, which is due to the very shallow nature of the excited state $0_{u}^{+}$ potential. The results for the line shifts from the close-coupled and perturbative calculations agree very closely at low laser intensities.
\end{abstract}

DOI: $10.1103 /$ PhysRevA.80.023417

PACS number(s): 34.50.Rk, 32.70.Jz, 34.50.Cx, 34.20.Cf

\section{INTRODUCTION}

Photoassociation (PA) experiments in which two interacting ultracold atoms (usually ground-state alkali-metal atoms or metastable rare-gas atoms) are resonantly excited by a laser to a molecular bound state provide a powerful technique to study the dynamics of ultracold collisions [1,2]. Since the colliding atoms are so cold, the energy of the initial scattering state is well determined and the resonant laser energies corresponding to transitions to various bound states produce a very high resolution spectrum $(<1 \mathrm{MHz})$. At this level of precision, energy level shifts induced by the PA laser can be significant [3-5] and an understanding of the dependence of the energy level shifts upon the laser intensity, polarization, and frequency is crucial.

The short-lived bound states created in photoassociation are unique in that they can occupy both the small interatomic regions of conventional molecules and the unusual large interatomic regions of hundreds of Bohr radii depending upon the particular intermolecular potential. For purely long-range molecules, the interaction between the atoms, and hence the molecular potentials, arises from dispersion forces that depend mainly upon well known atomic parameters. Extensions of the photoassociation technique can be used to determine the lifetimes of excited states [6], scattering lengths of ground states [7], create ground-state cold molecules [8], and drive other laser orientated processes.

Although ultracold physics and the achievement of BoseEinstein condensation has its roots in alkali-metal species, the cooling of excited state species such as metastable helium opens many more opportunities for experiment. Metastable rare-gas atoms offer exciting experimental detection strategies to study quantum gases as their large internal energy can be released through Penning and associative ionization during interatomic collisions and through ejection of electrons when the atoms strike a metal surface. Additionally, trap purity is more easily maintained in a metastable gas, as rare-gas ground states are not trapped by the same mecha- nisms as metastable states. The metastable atoms are generally spin-polarized in order to suppress the autoionization rate and to thereby attain large numbers of trapped atoms. As well, no hyperfine structure is present in rare-gas species making them more desirable to investigate than many other species.

In metastable helium a number of experimental investigations have been conducted using photoassociation as the diagnostic tool. Hershbach et al. [9] observed bound states that dissociate to the $2 s^{3} S_{1}+2 p^{3} P_{2}$ atomic limit, Léonard et al. [10] studied some purely long-range bound states with binding energies $\leq 1.43 \mathrm{GHz}$, dissociating to the $2 s^{3} S_{1}$ $+2 p{ }^{3} P_{0}$ limit, Kim et al. [11] and van Rijnbach [12] observed detailed structure of over 40 peaks associated with bound states with binding energies $\leq 13.57 \mathrm{GHz}$ that dissociate to the $2 s{ }^{3} S_{1}+2 p{ }^{3} P_{2}$ limit, and van Rijnbach [12] observed six peaks lying within $0.5 \mathrm{GHz}$ of the $2 s^{3} S_{1}$ $+2 p{ }^{3} P_{1}$ limit. Theoretical analyses of the long-range bound states dissociating to the $2 s{ }^{3} S_{1}+2 p{ }^{3} P_{0}$ limit have been completed using a single channel adiabatic calculation [13] and full multichannel calculations [14]. Both calculations use retarded long-range Born-Oppenheimer dispersion potentials and give excellent agreement with the measured binding energies. Most of the 40 peaks associated with the $2 s{ }^{3} S_{1}$ $+2 p{ }^{3} P_{2}$ limit were identified [15] using the accumulated phase technique for a single channel calculation of the bound states supported by a hybrid quintet potential constructed from short-range $a b$ initio ${ }^{5} \Sigma_{g / u}^{+}$and ${ }^{5} \Pi_{g / u}^{+}$potentials matched onto long-range retarded dispersion potentials. Recently Deguilhem et al. [16] have revisited the analysis of the PA peaks associated with the $2{ }^{3} S_{1}+2 p{ }^{3} P_{1,2}$ limits using fully $a b$ initio short-range potentials. Light-induced level shifts of several vibrational states in the long-range $J=1,0_{u}^{+}$potential associated with the $2 s{ }^{3} S_{1}+2 p{ }^{3} P_{0}$ asymptote have been measured by Kim et al. [17] and two-photon photoassociation spectroscopy has recently been used [18] to accurately measure the binding energy of the least bound vibrational level $(v=14)$ of the metastable ${ }^{5} \Sigma_{g}^{+}$state formed during the 
collision of two spin-polarized metastable helium atoms. The measured binding energy $E_{b}(v=14)=91.35 \pm 0.06 \mathrm{MHz}$, combined with the new ab initio ${ }^{5} \Sigma_{g}^{+}$potential of Przybytek and Jeziorski [19], yielded the high precision value $a$ $=7.512 \pm 0.005 \mathrm{~nm}$ for the $s$-wave scattering length.

All previous theoretical investigations of photoassociation involve limiting assumptions that may not be valid in the present investigation. Perturbative treatments of the radiative coupling [20,21] predict a linear dependence of the line shifts upon laser intensity but are only valid for low laser intensities. The analytical method of Simoni et al. [22] is valid for arbitrary laser intensities but assumes the radiative coupling vanishes asymptotically, thus avoiding the use of dressed states for the atoms in the radiation field. This is only valid when the coupling is negligible compared to the detuning to the atomic transition. The most detailed treatment is that of Napolitano [23] which employs a full multichannel treatment using dressed $s$ - and $d$-wave states. However, fine structure is neglected and it is assumed that the laser detunings are large compared to the radiative coupling.

Theoretical investigations of photoassociation in ultracold metastable helium are limited to the perturbative analysis of the light-induced energy level shifts of the excited $J=1,0_{u}^{+}$ rovibrational state [5] based upon the theories of [21,22]. In this model the shifts are linear in the laser intensity and in the $s$-wave scattering length $a$. However, as the $J=1,0_{u}^{+}$potential is very shallow, the laser detuning is quite small and the validity of not using dressed states needs investigation [24].

The goal of this paper is to present a complete treatment of photoassociation of spin-polarized metastable helium to the $j=1,0_{u}^{+}$state asymptoting to $2 s^{3} S_{1}+2 p{ }^{3} P_{0}$. This process is illustrated in Fig. 1. Of particular interest is the dependence of the photoassociation broadenings and level shifts upon laser intensity and polarization for small laser detunings comparable to the laser coupling.

This paper is organized as follows. The general formalism of two atoms colliding in the presence of a laser field is presented in Sec. II, a perturbative treatment of laser-induced energy level shifts in Sec. III, and a nonperturbative closecoupled treatment of the photoassociation resonance profile in Sec. IV. Section V presents our discussion and conclusions. Two appendixes provide more details on the basis states used in our calculations and the evaluation of the matrix elements of the system Hamiltonian in this basis. Atomic units are used in the actual calculations, with lengths in Bohr radii $a_{0}$ and energies in hartree $E_{h}=\alpha^{2} m_{e} c^{2}$ $=27.211396 \mathrm{eV}$.

\section{TWO-ATOM COLLISIONS IN A LIGHT FIELD}

\section{A. Hamiltonian}

The total Hamiltonian for two atoms colliding in the presence of a radiation field is

$$
\hat{H}=\hat{H}_{\mathrm{mol}}+\hat{H}_{\mathrm{rad}}+\hat{H}_{\mathrm{int}},
$$

where $\hat{H}_{\text {mol }}$ is the total molecular Hamiltonian (in barycentric coordinates)

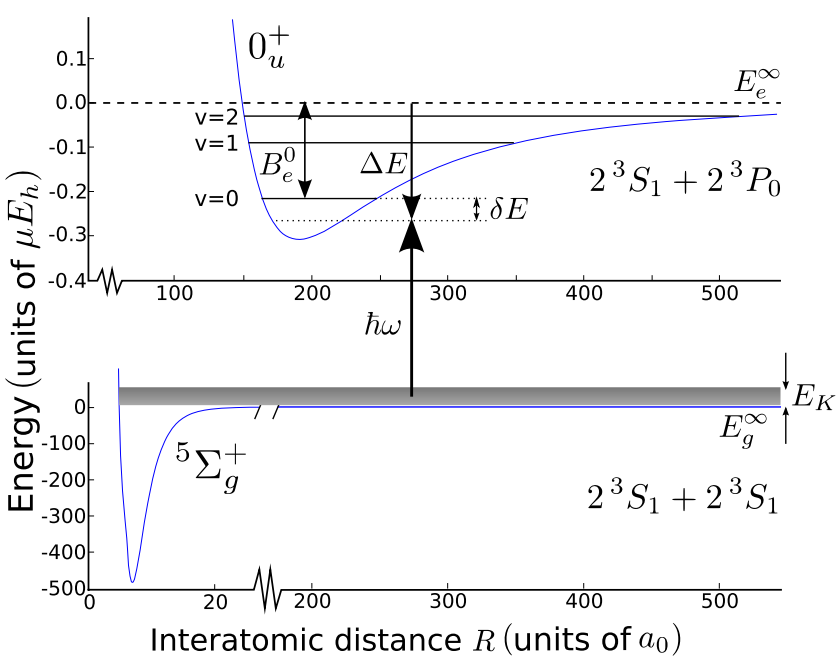

FIG. 1. (Color online) Photoassociation in metastable helium. Two spin-polarized metastable helium atoms $\left(2 s^{3} S_{1}+2 s{ }^{3} S_{1}\right)$ with very low kinetic energy $E_{K}$ absorb a photon of frequency $\omega$ and form a short-lived molecule in the rovibrational level $v$ of the $2 s{ }^{3} S_{1}+2 p{ }^{3} P_{0} O_{u}^{+}$excited state. The molecule can then spontaneously decay back to the disassociated metastable state (all other decay paths are spin-forbidden). As illustrated here for the $v=0$ level, $\delta E$ is the energy level shift induced by the laser field, $\Delta E$ is the laser detuning energy from the separated atom resonance, and $B_{e}^{0}$ is the binding energy of the level.

$$
\hat{H}_{\mathrm{mol}}=\hat{T}+\hat{H}_{\mathrm{rot}}+\hat{H}_{\mathrm{el}}+\hat{H}_{\mathrm{fs}} .
$$

Here $\hat{T}=-\left(\hbar^{2} / 2 \mu R\right)\left(\partial^{2} / \partial R^{2}\right) R$ is the kinetic energy operator, $\hat{H}_{\text {rot }}=\hat{l}^{2} /\left(2 \mu R^{2}\right)$ is the rotational operator for a system with relative angular momentum $\hat{l}$ and reduced mass $\mu, \hat{H}_{\mathrm{el}}=\hat{H}_{1}$ $+\hat{H}_{2}+\hat{H}_{12}$ is the total electronic Hamiltonian of the unperturbed atoms $\hat{H}_{1,2}$ and their electrostatic interaction $\hat{H}_{12}$, and $\hat{H}_{\mathrm{fs}}$ describes the fine structure of the atoms. The Hamiltonian for the free radiation field is $\hat{H}_{\mathrm{rad}}=\sum_{\xi} \hbar \omega_{\xi} \hat{a}_{\xi}^{\dagger} \hat{a}_{\xi}$ where $\hat{a}_{\xi}^{\dagger}\left(\hat{a}_{\xi}\right)$ are the usual creation (annihilation) operators for a photon of angular frequency $\omega_{\xi}$ and polarization $\boldsymbol{\epsilon}_{\xi}$ so that the field states are $\left|n, \omega_{\xi}, \boldsymbol{\epsilon}_{\xi}\right\rangle=(n !)^{-1 / 2}\left(\hat{a}_{\xi}^{\dagger}\right)^{n} \mid$ vac $\rangle$. The coupling between the two atoms and the radiation field is

$$
\hat{H}_{\mathrm{int}}=-\left(\frac{e}{m}\right) \sum_{i=1,2} \hat{\boldsymbol{p}}_{i} \cdot \hat{\boldsymbol{A}}\left(\boldsymbol{r}_{i}\right),
$$

where $\hat{\boldsymbol{p}}_{i}=-i \hbar \nabla_{\boldsymbol{r}_{i}}$. The vector potential is

$$
\hat{\boldsymbol{A}}\left(\boldsymbol{r}_{i}\right)=\sum_{\xi}\left[\boldsymbol{\varepsilon}_{\xi}\left(\boldsymbol{r}_{i}\right) \hat{a}_{\xi}+\boldsymbol{\varepsilon}_{\xi}\left(\boldsymbol{r}_{i}\right)^{*} \hat{a}_{\xi}^{\dagger}\right]
$$

where $\boldsymbol{\varepsilon}_{\xi}\left(\boldsymbol{r}_{i}\right)=\left(\hbar / 2 \omega_{\xi} \epsilon_{0} \mathcal{V}\right)^{1 / 2} \exp \left(i \boldsymbol{k} \cdot \boldsymbol{r}_{i}\right) \boldsymbol{\epsilon}_{\xi}$ and $\mathcal{V}$ is the normalization volume.

\section{B. Close-coupled equations}

The close-coupled equations describing the interaction of the two atoms in an applied laser field with given angular frequency $\omega$ and given polarization $\boldsymbol{\epsilon}_{\lambda}$ are obtained by expanding the energy eigenstates $|\Psi\rangle$ of $\hat{H}$ in terms of a basis of the form [25] 


$$
\left|\Phi_{a}, n\right\rangle \equiv\left|\Phi_{a}(R, q)\right\rangle \otimes\left|n, \omega, \boldsymbol{\epsilon}_{\lambda}\right\rangle,
$$

where $a$ denotes a set of approximate quantum numbers describing the electronic-rotational states of the molecule (to be discussed in Sec. II C), and $q$ denotes the interatomic polar coordinates $(\theta, \phi)$ and the electronic coordinates $\left(\boldsymbol{r}_{1}, \boldsymbol{r}_{2}\right)$. Using the expansion

$$
|\Psi\rangle=R^{-1}\left[\sum_{g^{\prime}} G_{g^{\prime}}(R)\left|\Phi_{g^{\prime}}, n\right\rangle+\sum_{e^{\prime}} G_{e^{\prime}}(R)\left|\Phi_{e^{\prime}}, n-1\right\rangle\right],
$$

where $g^{\prime}\left(e^{\prime}\right)$ labels the sets of metastable (excited) states, in $\hat{H}|\Psi\rangle=E|\Psi\rangle$ yields the close-coupled equations

$$
\begin{gathered}
\sum_{g^{\prime}}\left[T_{g g^{\prime}}^{G}+V_{g g^{\prime}} G_{g^{\prime}}(R)\right]+\sum_{e^{\prime}} V_{g e^{\prime}}^{\mathrm{int}} G_{e^{\prime}}(R)=(E-n \hbar \omega) G_{g}(R), \\
\sum_{e^{\prime}}\left[T_{e e^{\prime}}^{G}+V_{e e^{\prime}} G_{e^{\prime}}(R)\right]+\sum_{g^{\prime}} V_{e g^{\prime}}^{\mathrm{int}} G_{g^{\prime}}(R) \\
=[E-(n-1) \hbar \omega] G_{e}(R) .
\end{gathered}
$$

Here

$$
\begin{gathered}
T_{a^{\prime} a}^{G}=-\frac{\hbar^{2}}{2 \mu}\left\langle\Phi_{a^{\prime}}\left|\frac{\partial^{2}}{\partial R^{2}} G_{a}(R)\right| \Phi_{a}\right\rangle, \\
V_{a^{\prime} a}=\left\langle\Phi_{a^{\prime}}\left|\left[\hat{H}_{\mathrm{rot}}+\hat{H}_{\mathrm{el}}+\hat{H}_{\mathrm{fs}}\right]\right| \Phi_{a}\right\rangle,
\end{gathered}
$$

$(a=g$ or $e)$ and

$$
V_{e g}^{\mathrm{int}}=\left\langle\Phi_{e}, n-1\left|\hat{H}_{\mathrm{int}}\right| \Phi_{g}, n\right\rangle=\sqrt{\frac{I}{2 \epsilon_{0} c}}\left\langle\Phi_{e}\left|\boldsymbol{\epsilon}_{\lambda} \cdot \boldsymbol{d}\right| \Phi_{g}\right\rangle,
$$

where $I$ is the laser intensity and the molecular dipole operator $\boldsymbol{d}$ is the sum of the atomic dipole operators $\boldsymbol{d}_{i}=-e \boldsymbol{r}_{i}$. In obtaining Eq. (10) we have used [25]

$$
\left\langle\Phi_{e}\left|\frac{e}{m} \sum_{i} \hat{\boldsymbol{p}}_{i}\right| \Phi_{g}\right\rangle=\frac{i}{\hbar}\left\langle\Phi_{e}\left|\left[\hat{H}_{\mathrm{mol}}, \boldsymbol{d}\right]\right| \Phi_{g}\right\rangle,
$$

valid for the barycentric frame, and have invoked the dipole approximation $\exp \left(i \boldsymbol{k} \cdot \boldsymbol{r}_{i}\right) \approx 1$. This approximation has been used in other studies of photoassociation $[5,26]$ but does warrant some discussion. The outer turning points of the $J=1,0_{u}^{+}$ vibrational states considered here lie in the range $(250-470) a_{0}$, and $k=1 / 3258.17 a_{0}^{-1}$ for the $2 s^{3} S-2 p^{3} P$ transition so that the neglected next order term that would contribute is $\left(k r_{1,2}\right)^{2} \approx(k R / 2)^{2} \sim 5 \times 10^{-3}$, comparable to other errors in our calculation.

For photoassociation, the energy conservation relations are $E-n \hbar \omega=E_{g}^{\infty}+E_{K}$ and $E-(n-1) \hbar \omega=E_{e}^{\infty}-B_{e}^{v}+E_{K}+\hbar \Delta \omega$, where $E_{K}=\hbar^{2} k^{2} / 2 \mu$ is the kinetic energy of the colliding atoms, $E_{g, e}^{\infty}$ are the energies of the asymptotically free atoms, and $\Delta \omega=\omega-\omega_{0}$ is the laser detuning. Here $\hbar \omega_{0}=E_{e}^{\infty}-B_{e}^{v}$ $-E_{g}^{\infty}$ is the separation of the unperturbed excited and groundstate energies in the limit of zero kinetic energy.

\section{Basis states}

For two colliding atoms with orbital $\hat{\boldsymbol{L}}_{i}$, spin $\hat{\boldsymbol{S}}_{i}$, and total $\hat{\boldsymbol{j}}_{i}$ angular momenta, where $i=1,2$, several different basis representations can be constructed. Two possibilities are the $L S$ coupling scheme $\hat{\boldsymbol{L}}=\hat{\boldsymbol{L}}_{1}+\hat{\boldsymbol{L}}_{2}, \hat{\boldsymbol{S}}=\hat{\boldsymbol{S}}_{1}+\hat{\boldsymbol{S}}_{2}$, and $\hat{\boldsymbol{J}}=\hat{\boldsymbol{L}}+\hat{\boldsymbol{S}}+\hat{\boldsymbol{l}}$, and the $j j$ coupling scheme $\hat{\boldsymbol{j}}_{1}=\hat{\boldsymbol{L}}_{1}+\hat{\boldsymbol{S}}_{1}, \hat{\boldsymbol{j}}_{2}=\hat{\boldsymbol{L}}_{2}+\hat{\boldsymbol{S}}_{2}, \hat{\boldsymbol{j}}=\hat{\boldsymbol{j}}_{1}+\hat{\boldsymbol{j}}_{2}$, and $\hat{\boldsymbol{J}}=\hat{\boldsymbol{j}}+\hat{\boldsymbol{l}}$. The $L S$ coupling scheme diagonalizes $\hat{H}_{\mathrm{el}}$ whereas the $j j$ coupling scheme diagonalizes $\hat{H}_{\mathrm{fs}}$. As the magnitude of the fine-structure interaction is significantly larger than the electronic interaction at the long ranges of the photoassociated molecules, we use the $j j$ coupled states $\left|\gamma j_{1} j_{2} j l J m_{J}\right\rangle$ (see Appendix A), where $\gamma$ represents other relevant quantum numbers and $m_{J}$ labels the projections of the total angular momentum $\hat{\boldsymbol{J}}$ on to the space-fixed $O z$ axis. A further consideration is that the selection rules of the laser interaction refer to the space-fixed reference frame and couple states of differing $J$ and $m_{J}$, whereas the molecular interactions are more naturally described in the molecular reference frame in terms of $\Omega_{j}$, the projection of $j$ along the intermolecular axis $O Z$. Hence we choose the hybrid Hund case (c) molecular basis

$$
\left|\Phi_{a}(R, q)\right\rangle \equiv\left|\gamma_{1} \gamma_{2} j_{1} j_{2} j \Omega_{j} w, J m_{J}\right\rangle,
$$

where $w$ is the symmetry under inversion of the electronic wave function through the center of charge (see Appendix A). The matrix elements of the various contributions to the Hamiltonian in this basis are derived in Appendix B.

\section{PERTURBATIVE TREATMENT}

\section{A. F-operator technique}

For low laser intensities $\hat{H}_{\text {int }}$ can be treated as a perturbation. The unperturbed Hamiltonian is then $\hat{H}_{0}=\hat{H}_{\mathrm{mol}}+\hat{H}_{\mathrm{rad}}$ and has eigenstates of the form $R^{-1} G_{i}^{0}(R)\left|\psi_{i}^{0}\right\rangle \otimes\left|n, \omega, \boldsymbol{\epsilon}_{\lambda}\right\rangle$ where the unperturbed radial functions $G_{i}^{0}(R)$ satisfy Eq. (7) with $V_{e g}^{\text {int }}$ omitted and $E$ replaced by the total unperturbed energy $E_{0}$.

To calculate the second-order laser-induced energy level shifts $\delta E_{e}^{(2)}$ of the excited bound states $|e\rangle$ we note that [14] have shown that the adiabatic approximation has little effect on the bound levels making it possible to consider each excited state as an isolated state. The adiabatic states are formed by diagonalizing $\hat{H}_{\text {mol }}$ without the radial kinetic term and can be written as

$$
\left|\psi_{e}^{0}\right\rangle=\sum_{a} C_{e a}(R)\left|\Phi_{a}\right\rangle
$$

where $\Sigma_{a}\left|C_{e a}(R)\right|^{2}=1$.

We employ the $F$-operator technique $[27,28]$ to evaluate $\delta E_{e}^{(2)}$. This approach has recently been used by Beams et al. $[29,30]$ to treat spin-dipole interactions as a perturbation and we adapt their formalism here to the laser-matter interaction $\hat{H}_{\text {int }}$. The shift is given by 


$$
\delta E_{e}^{(2)}=\left\langle e\left|\hat{H}_{\mathrm{int}} \hat{F}\right| e\right\rangle-\langle e|\hat{F}| e\rangle\left\langle e\left|\hat{H}_{\mathrm{int}}\right| e\right\rangle=\left\langle e\left|\hat{H}_{\mathrm{int}} \hat{F}\right| e\right\rangle,
$$

where the second term is zero as $\left\langle e\left|\hat{H}_{\text {int }}\right| e\right\rangle$ vanishes due to dipole selection rules. The operator $\hat{F}$ satisfies

$$
\left[\hat{F}, \hat{H}_{0}\right]|e\rangle=\hat{H}_{\text {int }}|e\rangle .
$$

We assume the states $\left\{\left|\Phi_{g^{\prime}}, n\right\rangle\right\}$ and $\left|\psi_{e}^{0}, n-1\right\rangle \equiv\left|\psi_{e}^{0}\right\rangle$ $\otimes\left|n-1, \omega, \boldsymbol{\epsilon}_{\lambda}\right\rangle$ form a basis and expand $\hat{F}|e\rangle$ in terms of them:

$$
\hat{F}|e\rangle=R^{-1}\left[\sum_{g^{\prime}} f_{g^{\prime}}(R)\left|\Phi_{g^{\prime}}, n\right\rangle+f_{e^{\prime}}(R)\left|\psi_{e^{\prime}}^{0}, n-1\right\rangle\right],
$$

so that Eq. (15) gives the coupled equations

$$
\left[\frac{\hbar^{2}}{2 \mu} \frac{d^{2}}{d R^{2}}+\left[E_{0}-(n-1) \hbar \omega\right]-V_{e}^{\mathrm{KC}}-0_{u}^{+}(R)\right] f_{e}(R)=0
$$

and

$$
\begin{aligned}
& {\left[\frac{\hbar^{2}}{2 \mu} \frac{d^{2}}{d R^{2}}+\left(E_{0}-n \hbar \omega\right)\right] f_{g^{\prime}}(R)-\sum_{g}\left[V_{g^{\prime} g}^{\mathrm{rot}}+V_{g^{\prime} g}^{\mathrm{el}}\right] f_{g}(R)} \\
& \quad=G_{e}^{0}(R) V_{g^{\prime}}^{\mathrm{int}}(R) .
\end{aligned}
$$

We have assumed no $R$ dependence of the basis states $\left|\Phi_{a}\right\rangle$ but include the correction term $V_{e}^{\mathrm{KC}}$ for the excited state (see Appendix B). The matrix elements are

$$
\begin{aligned}
& V_{g^{\prime} g}^{\mathrm{el}}=\left\langle\Phi_{g^{\prime}}\left|\hat{H}_{\mathrm{el}}\right| \Phi_{g}\right\rangle, \\
& V_{g^{\prime} g}^{\mathrm{rot}}=\left\langle\Phi_{g^{\prime}}\left|\hat{H}_{\mathrm{rot}}\right| \Phi_{g}\right\rangle
\end{aligned}
$$

and $0_{u}^{+}(R)$ is the adiabatic potential of the excited state:

$$
0_{u}^{+}(R)=\left\langle\psi_{e}^{0}\left|\left[\hat{H}_{\mathrm{rot}}+\hat{H}_{\mathrm{el}}+\hat{H}_{\mathrm{fs}}\right]\right| \psi_{e}^{0}\right\rangle .
$$

The interaction term is

$$
V_{g}^{\mathrm{int}}(R)=\left\langle\Phi_{g}, n\left|\hat{H}_{\text {int }}\right| \psi_{e}^{0}, n-1\right\rangle
$$

and $G_{e}^{0}(R)$ is the radial eigenfunction for the rovibrational level $v$ of the excited state.

Substitution of expansion (16) into Eq. (14) yields

$$
\delta E_{e}^{(2)}=\sum_{g} \int d R G_{e}^{0}(R) V_{g}^{\mathrm{int}}(R) f_{g}(R) .
$$

Consequently, we need only solve the inhomogeneous equations (18). These are solved using a discrete-variable representation method [31] with a cosine Fourier basis. As the laser interaction region requires closely spaced grid points and the asymptotic region very few points, a scaled grid $R$ $=\zeta(t)[29]$ was used where the mesh in $t$ is equispaced. A quartic scaling was chosen as it does not modify the boundary conditions.

\section{B. Application to metastable helium}

We consider photoassociation to an excited long-range bound level in the $J=1, m_{J}=1,0_{u}^{+}$adiabatic potential that asymptotes to a $\mathrm{He}\left(2 s^{3} S_{1}\right)+\mathrm{He}\left(2 p^{3} P_{0}\right)$ diatom (see Fig. 1). The adiabatic state can be expressed as a combination of the four basis states $\left|j_{2}, j\right\rangle$,

$$
|0,1\rangle|1,1\rangle|2,1\rangle|2,3\rangle,
$$

where we have suppressed the common quantum numbers $\gamma_{1}=\left(L_{1}, S_{1}\right)=(0,1), \gamma_{2}=\left(L_{2}, S_{2}\right)=(1,1), j_{1}=1, \Omega_{j}=0, J=m_{J}$ $=1$, and $w=1$. Coriolis couplings and the off-diagonal kinetic terms (see Appendix B) have been ignored, as their effect has been shown to be small at long range [14].

An analysis of $\hat{H}_{\text {int }}$ shows that only a small manifold of metastable basis states are coupled to the excited state. Using the reduced state notation of $\left|j \Omega_{j}, J m_{J}\right\rangle$, the states coupled by $\sigma^{-}$laser light are

$$
\begin{gathered}
|00,22\rangle,|20,22\rangle,|21,22\rangle,|2-1,22\rangle, \\
(|22,22\rangle,|2-2,22\rangle)
\end{gathered}
$$

whereas the states coupled by $\sigma^{+}$laser light are

$$
\begin{gathered}
|00,00\rangle,|00,20\rangle,|20,00\rangle,|20,20\rangle, \\
|21,10\rangle,|2-1,10\rangle,|21,20\rangle,|2-1,20\rangle, \\
(|20,10\rangle,|22,20\rangle,|2-2,20\rangle) .
\end{gathered}
$$

The bracketed states are coupled by the Coriolis terms of $\hat{H}_{\text {rot }}$ to other metastable states and hence are indirectly coupled to the excited state. The implicit quantum numbers for these metastable states are $\gamma_{1}=\left(L_{1}, S_{1}\right)=(0,1), \gamma_{2}=\left(L_{2}, S_{2}\right)=(0,1)$, $j_{1}=j_{2}=1$, and $w=0$.

The calculations require Born-Oppenheimer potentials for the ${ }^{5} \Sigma_{g}^{+}$and ${ }^{1} \Sigma_{g}^{+}$states of the two metastable $\mathrm{He}\left(2 s^{3} S_{1}\right)$ atoms and the $J=1,0_{u}^{+}$potential of the excited $\operatorname{He}\left(2 s^{3} S_{1}\right)$ $+\mathrm{He}\left(2 p^{3} P_{0}\right)$ system. For the quintet potential ${ }^{5} \Sigma_{g}^{+}$we use that given by Przybytek and Jeziorski [19], which includes adiabatic, relativistic, and QED corrections. For the singlet potential ${ }^{1} \Sigma_{g}^{+}$we use [29] a potential constructed from the short-range Müller et al. [32] potential exponentially damped onto the quintet potential at long range. The excited state potentials are constructed from the 12 Born-Oppenheimer dispersion potentials [14] $f_{3 \Lambda}(R / \chi) C_{3 \Lambda} / R^{3}+C_{6 \Lambda} / R^{6}$ $+C_{8 \Lambda}^{ \pm} / R^{8}$, where $f_{3 \Lambda}$ is an $R$ - and $\Lambda$-dependent retardation correction [33], $\lambda=3258.17 a_{0}$, where $\lambda=\lambda /(2 \pi)$ and $\lambda$ is the $2 s^{3} S-2 p^{3} P$ transition wavelength. In particular, the excited state $\left|0_{u}^{+}\right\rangle$is a linear combination of the ${ }^{5} \Sigma_{u}^{+},{ }^{5} \Pi_{u},{ }^{3} \Pi_{u}$, and ${ }^{1} \Sigma_{u}^{+}$states. The $C_{3 \Sigma}$ coefficient is $\pm 2 C_{3}$ and $C_{3 \Pi}$ is $\pm C_{3}$, where $C_{3}=6.41022 E_{h} a_{0}^{3}$ and is proportional to the square of the $2 s^{3} S-2 p^{3} P$ transition dipole matrix element. For the van der Waals coefficients we use $C_{6 \Sigma}=2620.76 E_{h} a_{0}^{6}$ and $C_{6 \Pi}$ $=1846.60 E_{h} a_{0}^{6}$. The $C_{8 \Lambda}^{ \pm}$terms are [34] $C_{8 \Sigma}^{+}=151383 E_{h} a_{0}^{8}$, $C_{8 \Sigma}^{-}=297215.9 E_{h} a_{0}^{8}, \quad C_{8 \Pi}^{+}=97244.75 E_{h} a_{0}^{8}, \quad$ and $C_{8 \Pi}^{-}$ $=162763.8 E_{h} a_{0}^{8}$. Here the superscript indicates the sign of $(-1)^{S+w}$ where $S$ is the spin of the state and $w=0(1)$ for gerade (ungerade) symmetry. The $C_{3} / R^{3}$ term is the domi- 
TABLE I. Perturbative line shifts per laser intensity in units of $\mathrm{MHz} /\left(\mathrm{W} \mathrm{cm}^{-2}\right)$ for the long-range bound states of the $\mathrm{He}\left(2{ }^{3} S_{1}\right)$ $+\mathrm{He}\left(2 p^{3} P_{0}\right), 0_{u}^{+}$configuration. Results are given for calculations without and with the correction $V_{e}^{\mathrm{KC}}$.

\begin{tabular}{ccccc}
\hline \hline Level & Polarization & No $V_{e}^{\mathrm{KC}}$ & With $V_{e}^{\mathrm{KC}}$ & Ref. [5] \\
\hline$v=0$ & $\sigma^{-}$ & -6.439 & -6.507 & -6.37 \\
& $\sigma^{+}$ & -7.724 & -7.784 & -7.36 \\
$v=1$ & $\sigma^{-}$ & -11.662 & -11.748 & -11.70 \\
& $\sigma^{+}$ & -10.205 & -10.270 & -10.25 \\
$v=2$ & $\sigma^{-}$ & -29.442 & -29.692 & -29.57 \\
& $\sigma^{+}$ & -24.675 & -24.877 & -24.11 \\
\hline \hline
\end{tabular}

nant contribution to the dispersion potential for the purelylong-range states. Also required is the $2 s \rightarrow 2 p$ atomic dipole moment $d_{\mathrm{at}}^{\mathrm{sp}}=2.14583 \times 10^{-29} \mathrm{C} \mathrm{m}$ and the fine-structure splittings. For He the $2 p{ }^{3} P_{0}$ and $2 p{ }^{3} P_{1}$ levels lie 31.9081 $\mathrm{GHz}$ and $2.2912 \mathrm{GHz}$ above the $2{ }^{3} P_{2}$ level, respectively.

The results for the three lowest vibrational bound levels and for laser polarizations $\sigma^{ \pm}$are given in Table I. In order to compare with the results of Portier et al. [5], we have used $\Delta \omega=0$, corresponding to a zero kinetic energy. There is good general agreement between the two sets of results with the small differences probably due to the potentials used.

\section{CLOSE-COUPLED CALCULATION OF PHOTOASSOCIATION PROFILE}

\section{A. Hamiltonian matrix}

The close-coupled calculation is nonperturbative in that differential equations (7) are solved without assuming the laser interaction is weak. In this approach the scattering matrix elements are calculated and the PA profile obtained for various laser detunings, intensities, and temperatures.

Although the method presented here is quite general, for ease of visualization we explicitly formulate it for photoassociation in metastable helium of the subset of states coupled by $\sigma^{-}$polarized light in Eq. (24). Specifically these states $|\alpha\rangle$ are

$$
\begin{gathered}
|1\rangle=\left|j=0, \Omega_{j}=0, J=2, m_{J}=2\right\rangle \otimes\left|n, \omega, \boldsymbol{\epsilon}_{\lambda}\right\rangle, \\
|2\rangle=\left|j=2, \Omega_{j}=-2, J=2, m_{J}=2\right\rangle \otimes\left|n, \omega, \boldsymbol{\epsilon}_{\lambda}\right\rangle, \\
|3\rangle=\left|j=2, \Omega_{j}=-1, J=2, m_{J}=2\right\rangle \otimes\left|n, \omega, \boldsymbol{\epsilon}_{\lambda}\right\rangle, \\
|4\rangle=\left|j=2, \Omega_{j}=0, J=2, m_{J}=2\right\rangle \otimes\left|n, \omega, \boldsymbol{\epsilon}_{\lambda}\right\rangle, \\
|5\rangle=\left|j=2, \Omega_{j}=1, J=2, m_{J}=2\right\rangle \otimes\left|n, \omega, \boldsymbol{\epsilon}_{\lambda}\right\rangle, \\
|6\rangle=\left|j=2, \Omega_{j}=2, J=2, m_{J}=2\right\rangle \otimes\left|n, \omega, \boldsymbol{\epsilon}_{\lambda}\right\rangle, \\
|7\rangle=\left|0_{u}^{+}, J=1, m_{J}=1\right\rangle \otimes\left|n-1, \omega, \boldsymbol{\epsilon}_{\lambda}\right\rangle,
\end{gathered}
$$

for which the close-coupled equations (7) reduce to

$$
\sum_{\alpha^{\prime}}\left[-\frac{\hbar^{2}}{2 \mu} \frac{d^{2}}{d R^{2}} \delta_{\alpha \alpha^{\prime}}+W_{\alpha \alpha^{\prime}}(R)\right] G_{\alpha^{\prime}}(R)=0,
$$

where the potential matrix $\boldsymbol{W}$ is

$$
\boldsymbol{W}=\left[\begin{array}{ccccccc}
V_{1}^{0}-E_{K} & 0 & 0 & 0 & 0 & 0 & \hbar \Omega_{1}^{0} \\
0 & V_{5}^{-2}-E_{K} & L^{12} & 0 & 0 & 0 & 0 \\
0 & L^{12} & V_{5}^{-1}-E_{K} & L^{01} & 0 & 0 & \hbar \Omega_{5}^{-1} \\
0 & 0 & L^{01} & V_{5}^{0}-E_{K} & L^{01} & 0 & \hbar \Omega_{5}^{0} \\
0 & 0 & 0 & L^{01} & V_{5}^{1}-E_{K} & L^{12} & \hbar \Omega_{5}^{1} \\
0 & 0 & 0 & 0 & L^{12} & V_{5}^{2}-E_{K} & 0 \\
\hbar \Omega_{1}^{0} & 0 & \hbar \Omega_{5}^{-1} & \hbar \Omega_{5}^{0} & \hbar \Omega_{5}^{1} & 0 & V_{e}+V_{e}^{\mathrm{KC}}-\Delta E
\end{array}\right]
$$

Here

$$
V_{2 j+1}^{\Omega_{j}}={ }^{2 j+1} \Sigma_{g}^{+}(R)+\left\langle j \Omega_{j}\left|\hat{H}_{\mathrm{rot}}\right| j \Omega_{j}\right\rangle
$$

is the effective ground-state potential comprising the BornOppenheimer potential for the ${ }^{2 j+1} \Sigma_{g}^{+}$state and the diagonal rotational couplings, $V_{e}=0_{u}^{+}(R)-i \Gamma / 2$ is the adiabatic potential of the excited state, and $\Delta E=\hbar \Delta \omega+E_{K}-B_{e}^{v}$. The offdiagonal terms are the Coriolis couplings

$$
L^{\Omega_{j}, \Omega_{j}^{\prime}}=\left\langle j=2, \Omega_{j}^{\prime}\left|\hat{H}_{\text {rot }}\right| j=2, \Omega_{j}\right\rangle
$$

and the radiative couplings $\hbar \Omega_{2 j+1}^{\Omega_{j}}=V_{g}^{\text {int }}(R)$ expressed in terms of the Rabi frequencies $\Omega_{2 j+1}^{\Omega_{j}}$.

The decay of the excited state through spontaneous emission is represented by the molecular width $\Gamma=\Gamma\left(R / \chi, \Gamma_{\mathrm{at}}\right)$ where the $R$ dependence arises from the retarded dipole interaction expressed in the Hund's case (a) states [33] and its subsequent diagonalization as part of the formation of the adiabatic potential. For our ungerade system, $\Gamma \approx 2 \Gamma_{\text {at }}$ for the 
interaction region and $\Gamma \approx \Gamma_{\text {at }}$ for the asymptotic region $R$ $\gg \chi$. The spontaneous emission atomic line width for the triplet helium $2 s-2 p$ transition is $\Gamma_{\text {at }}=1.626 \mathrm{MHz}$. Note that the asymptotic energies $E_{g, e}^{\infty}$ cancel in Eq. (28).

The definition of scattering matrix elements requires asymptotically free states. However, the asymptotic form $\boldsymbol{W}_{\infty}$ of $\boldsymbol{W}$ is not diagonal as the Rabi couplings do not vanish at large $R$ and the separated atoms remained coupled by the laser interaction. Two options are available to determine the $S$-matrix elements: (i) transformation from the basis states $|\alpha\rangle$ to the dressed-state basis states [25] $|\beta\rangle=\Sigma_{\alpha} U_{\beta \alpha}|\alpha\rangle$ in which $\boldsymbol{W}_{\infty}^{\mathrm{D}}=\boldsymbol{U}^{-1} \boldsymbol{W}_{\infty} \boldsymbol{U}$ is diagonal or (ii) introduction of a modified radiative coupling for $R$ greater than some large value $R_{z}$ :

$$
\tilde{V}_{e g}^{\mathrm{int}}(R)= \begin{cases}V_{e g}^{\mathrm{int}}(R) & \text { for } R \leq R_{z} \\ V_{e g}^{\mathrm{int}}(R) \exp \left[-\rho\left(R-R_{z}\right)^{2}\right] & \text { for } R>R_{z} .\end{cases}
$$

This coupling vanishes asymptotically and simulates the experimental conditions of the laser being switched off before and after the experiment.

\section{B. Dressed states}

Napolitano [23] has considered the case of six dressed states with laser couplings restricted to two pairs of states and has obtained analytical results valid for large red detunings $\Delta E \gg \Gamma$ and $\Delta E \gg \Omega_{5}^{\Omega_{j}}$. As we wish to avoid such assumptions and also develop a procedure valid for a larger number of coupled states, we use direct numerical diagonalization.

In terms of dressed states the expansion of the state vector becomes

$$
|\Psi\rangle=R^{-1} \sum_{\alpha} G_{\alpha}(R)|\alpha\rangle=R^{-1} \sum_{\beta} \widetilde{G}_{\beta}(R)|\beta\rangle,
$$

where the dressed-state radial amplitudes satisfy

$$
\sum_{\beta^{\prime}}\left[-\frac{\hbar^{2}}{2 \mu} \frac{d^{2}}{d R^{2}} \delta_{\beta \beta^{\prime}}+W_{\beta \beta^{\prime}}^{\mathrm{D}}(R)\right] \widetilde{G}_{\beta^{\prime}}(R)=0
$$

and $\boldsymbol{W}^{\mathrm{D}}=\boldsymbol{U}^{-1} \boldsymbol{W} \boldsymbol{U}$. Asymptotically the dressed states decouple and satisfy

$$
\left[\frac{d^{2}}{d R^{2}}+\frac{2 \mu E_{\beta}}{\hbar^{2}}\right] \widetilde{G}_{\beta}(R)=0,
$$

where the energies $E_{\beta} \equiv-W_{\beta \beta}^{\mathrm{D}}(R=\infty)$ can be complex. Defining $k_{\beta}=\sqrt{2 \mu E_{\beta}} / \hbar=k_{\beta}^{r}+i k_{\beta}^{i}$, the asymptotic solutions of Eq. (34) have the form

$$
\widetilde{G}_{\beta}(R) \sim c_{1} e^{-k_{\beta}^{i} R} e^{i k_{\beta}^{r} R}+c_{2} e^{k_{\beta}^{i} R} e^{-i k_{\beta}^{r} R} .
$$

The open-channel and closed-channel manifolds are then identified, respectively, as those states that persist or vanish as $R \rightarrow \infty$. Therefore, we require $k_{\beta}^{i}$ to be zero for the open channels.

After diagonalization we find that two of the energies $E_{\beta}$ are nondegenerate and their associated dressed states include undressed excited state contributions. The third value of $E_{\beta}$ is purely real, fivefold degenerate, and mixes only the undressed open channels for the ground states. As the diagonalization is numerical, the particular combination of ground states in this degenerate subspace is dependent upon the numerical procedure used and the combinations will not vary smoothly as the detuning is changed. However, the $S$-matrix elements that involve only the states with nondegenerate energies will be guaranteed to vary smoothly.

For the orthogonal polarization $\sigma^{+}$we briefly note that the above analysis applied to the 11 metastable states listed in Eq. (25) exhibits very similar behavior. Three unique values for $E_{\beta}$ are observed, two that are nondegenerate and one that is tenfold degenerate. No differences in the analysis or numerical implementation apply other than to include a larger number of states in the $\sigma^{+}$system.

\section{Modified radiation coupling}

Because the dressed states introduce complex asymptotic energies, the simplicity offered by using a modified radiative coupling becomes more attractive. To implement the modified coupling, we use the undressed form of state vector (32) to obtain

$$
\sum_{\alpha^{\prime}}\left[-\frac{\hbar^{2}}{2 \mu} \frac{d^{2}}{d R^{2}} \delta_{\alpha \alpha^{\prime}}+W_{\alpha \alpha^{\prime}}^{z}(R)\right] \widetilde{G}_{\alpha^{\prime}}(R)=0,
$$

where $W_{\alpha \alpha^{\prime}}^{z}$ is identical to $W_{\alpha \alpha^{\prime}}$ except that $V_{e g}^{\text {int }}$ is replaced by $\tilde{V}_{e g}^{\text {int }}$. This system consists of six open channels, all with identical values of $k_{\alpha}=\sqrt{2 \mu E_{K}} / \hbar$ and one closed channel.

\section{Extraction of $S$-matrix elements}

The $S$ matrix is determined by matching the asymptotic solutions of Eq. (33) or Eq. (36) to the combination [35]

$$
\widetilde{\boldsymbol{G}}=\boldsymbol{H}_{-}^{0} \boldsymbol{A}+\boldsymbol{H}_{+}^{0} \boldsymbol{B}=\left(\boldsymbol{H}_{-}^{0}-\boldsymbol{H}_{+}^{0} \boldsymbol{S}\right) \boldsymbol{A},
$$

where $\widetilde{G}_{\gamma \gamma^{\prime}}$ is the matrix of solutions formed from $\widetilde{G}_{\gamma}(R)$ with the second subscript $\gamma^{\prime}$ labeling the linearly independent solutions generated by different choices of boundary conditions. We have introduced the notation $\gamma=\beta$ for the dressed states approach and $\gamma=\alpha$ for the modified coupling approach. The diagonal matrices $\left(H_{ \pm}^{0}\right)_{\gamma \gamma^{\prime}}=\delta_{\gamma \gamma^{\prime}} h_{\gamma}^{ \pm}$for the open channel scattering states have the asymptotic form of outward and inward traveling waves,

$$
h_{\gamma}^{ \pm} \underset{R \rightarrow \infty}{\sim}\left(2\left|k_{\gamma}\right|\right)^{-1 / 2} e^{ \pm i k_{\gamma} R},
$$

whereas for the closed channels the asymptotic forms are

$$
h_{\gamma}^{ \pm} \underset{R \rightarrow \infty}{\sim}\left(2\left|k_{\gamma}\right|\right)^{-1 / 2} e^{\mp\left|k_{\gamma}\right| R} .
$$

The matrices $\boldsymbol{A}, \boldsymbol{B}$, and $\boldsymbol{S}=-\boldsymbol{B} \boldsymbol{A}^{-1}$ have the structure

$$
\boldsymbol{A}=\left[\begin{array}{ll}
\boldsymbol{A}_{o o} & \boldsymbol{A}_{o c} \\
\boldsymbol{A}_{c o} & \boldsymbol{A}_{c c}
\end{array}\right],
$$

where the labels $o$ and $c$ refer, respectively, to open and closed channels. We require only the open-open contribu- 
tions $\boldsymbol{S}_{o o}$ which, as we can enforce $\boldsymbol{A}_{c o}=0$ by careful choice of boundary conditions, are given by $\boldsymbol{S}_{o o}=-\boldsymbol{B}_{o o} \boldsymbol{A}_{o o}^{-1}$.

As $E_{\beta}=E_{\beta}^{r}+i E_{\beta}^{i}$ and $k_{\beta}$ can be complex for the dressed states, the asymptotic solutions do not have the usual plane wave oscillatory form and care must be taken during the matching process. In the present case $E_{\beta}$ is complex for only two of the dressed states. One is clearly a closed channel as it has $E_{\beta}^{r}<0$. The other has $E_{\beta}^{r}>0$ and a relatively small value of $E_{\beta}^{i}$. Without the imaginary component, this channel would have a purely real value of $k_{\beta}$ and be considered an open channel. However, as $k_{\beta}$ is complex, the state has the asymptotic form (35) consisting of exponentially increasing and decreasing solutions. To treat the solution rigorously, we must enforce finiteness by discarding the exponentially increasing solution and integrate out to a sufficiently large value of $R$ that the exponentially decreasing solution has completely dampened indicating that the channel is closed. The $S$ matrix can then be created from the remaining open channels that have purely real $E_{\beta}$ components. The difficulty with such an approach is that $k_{\beta}^{r} \gg k_{\beta}^{i}$ requiring the integration range to be very large imposing a heavy computational burden. Note that $k_{\beta}^{r}$ and $k_{\beta}^{i}$ will also vary with the laser detuning and laser intensity, further complicating the problem.

An alternative approach is to consider the problematic channel as a pseudo-open channel. By redefining the $S$ matrix, such that the asymptotic functions are matched at a finite distance $R=R_{\max }$ instead of $R \rightarrow \infty$, we can ensure the pseudo-open channel has a finite inward and outward flux. This is performed by treating the terms $e^{\mp k_{\beta}^{i} R}$ as approximately constant in the local region of $R_{\max }$ and matching to the oscillatory behavior of $e^{ \pm i k_{\beta}^{r} R}$. This type of matching obviously has a dependence upon the matching point but we expect it to not vary the shape of the profile significantly if $R_{\max }$ is chosen outside the interaction region. The resultant $S$-matrix element $S_{\beta^{\prime} \beta}$ then gives the probability that the system with unit flux in an incoming channel $|\beta\rangle$ at $R_{\max }$ makes a transition to an outgoing channel $\left|\beta^{\prime}\right\rangle$ at $R_{\max }$. We note that this choice of $R_{\max }$ bears some similarity to the choice of $R_{z}$ for the modified coupling method.

\section{E. Detection of the resonance}

The photoassociation resonance can be studied by analyzing the loss from the excited state due to spontaneous emission so that the photoassociation line shape is due to the emitted photons and therefore proportional to the loss of unitarity of the $S$ matrix. For atoms colliding in the entrance channel $|\gamma\rangle$, the loss rate is $\mathcal{L}_{\gamma}=\left\langle v_{\gamma} \sigma_{\gamma}^{\text {photon }}\right\rangle$ where the cross section for photon emission is

$$
\sigma_{\gamma}^{\text {photon }}=\frac{\pi}{k_{\gamma}^{2}}\left(1-\sum_{\gamma^{\prime}}\left|S_{\gamma^{\prime} \gamma}\right|^{2}\right)
$$

and $\langle\cdots\rangle$ denotes a thermal average over a distribution of the asymptotic relative velocities $v_{\gamma}=\hbar k_{\gamma} / \mu$ of the two colliding atoms. For temperatures of order $1 \mu \mathrm{K}$ this thermal averaging can be ignored. The energy dependence of $\sigma_{\gamma}^{\text {photon }}$ is that of a peak superimposed upon a slowly varying background and can be well fitted in the region of the peak by a Fano profile [36] of the form

$$
\sigma_{\gamma}^{\text {photon }}=A_{\text {bg }}(\epsilon)-A_{\text {res }} \frac{(\epsilon+q)^{2}}{1+\epsilon^{2}},
$$

where $A_{\mathrm{bg}}(\epsilon)$ describes a linear background, $A_{\text {res }}$ is a constant, $\epsilon=\left(E-E_{\text {res }}\right) /\left(\Gamma_{\text {res }} / 2\right)$ is a normalized energy, and $E_{\text {res }}$ and $\Gamma_{\text {res }}$ are the position and full width of the resonance. The Fano parameter $q$ is a measure of the ratio of the direct (background) to resonant scattering. As we often deal with Lorentzian-type behavior that occurs in the limit $q \rightarrow \infty$ and $A_{\text {res }} \rightarrow 0$, it is simpler numerically to instead match to the form

$$
\sigma_{\gamma}^{\text {photon }}=A_{\text {bg }}(\epsilon)-A_{\text {res }}^{\prime} \frac{(1+p \epsilon)^{2}}{1+\epsilon^{2}},
$$

where $p=1 / q$ and $A_{\text {res }}^{\prime}=q A_{\text {res }}$. For the present calculations we find $p \lesssim 10^{-2}$ except for the dressed-state profiles at high intensity where $p \sim 0.5$.

\section{F. Numerical issues}

To solve the dressed problem, the undressed asymptotic potential matrix $\boldsymbol{W}_{\infty}$ is diagonalized by a Hermitian eigendecomposition. The renormalized Numerov [37] boundary value method is then used to integrate either the dressed equations (33) or the modified coupling equations (36). The outer boundary used is either $R_{\max }$ for the dressed states or, for the modified coupling, a value greater than $R_{z}$ such that the radiation coupling has been completely turned off. To ensure the asymptotic solutions are uncoupled, we choose $R_{\max }$ and $R_{z}$ to be greater than $10^{4} a_{0}$. Typically we use $R_{z}$ $=2 \times 10^{5} a_{0}$ and $\rho \sim O\left(5 k_{\gamma}^{-1}\right)=4 \times 10^{-6} a_{0}^{-1}$. The linearly independent solutions $\tilde{G}_{\gamma \gamma^{\prime}}, \gamma^{\prime}=1, \ldots, \mathcal{N}-1$, are generated by choosing $\mathcal{N}-1$ linearly independent boundary conditions.

A kinetic energy of $10^{-11} E_{h}(2.1 \mu \mathrm{K})$ is chosen, which places the system just above temperatures for which recent experiments have reported Bose-Einstein condensation and which does not introduce a prohibitively large outer boundary for the numerical integration. Note that quantitatively, at an intensity of $0.7 \mathrm{~W} / \mathrm{cm}^{2}$, the matrix elements in $\boldsymbol{W}_{\infty}$ are $O\left(10^{-11}\right)$ and $O\left(10^{-7}\right)$ for the diagonal elements $E_{K}$ and $\Delta E$, respectively, and $O\left(10^{-9}\right)$ for the off-diagonal Rabi couplings $\Omega_{2 j+1}^{\Omega_{j}}$.

\section{G. Results}

We first consider the results for low to moderate laser intensities as they exhibit behavior similar to regular spectroscopic profiles. As the laser intensity is increased, unusual aspects of the dressed and modified coupling become apparent and we shall discuss these separately.

The central positions and broadenings of the dressed PA profiles were determined from the fits to Fano profiles (42) for the cross section $\sigma_{\gamma}^{\text {photon }}$ as a function of laser energy. For nondegenerate channels $|\beta\rangle$, such as the pseudo-open channel in the present investigation, the variation in $\sigma_{\beta}^{\text {photon }}$ with laser energy is smooth. However, as previously mentioned, 


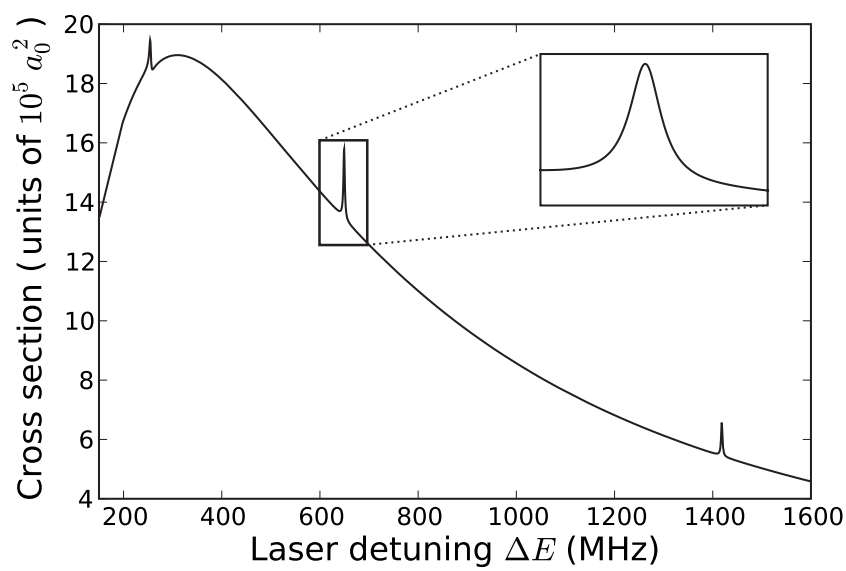

FIG. 2. PA cross-section profile calculated using dressed states. Results shown are for $\sigma^{-}$polarization and a low intensity of $64 \mathrm{~mW} / \mathrm{cm}^{2}$.

the numerical diagonalization process arbitrarily selects the degenerate dressed states $\left\{\left|\beta_{d}\right\rangle\right\}$ in the subspace $\mathcal{E}_{d}$ that they span. This means that $\sigma_{\beta}^{\text {photon }}$ for any $\beta \in \beta_{d}$ will not vary smoothly with laser energy.

Fortunately, for these degenerate dressed channels we are permitted to analyze various combinations of $\sigma_{\beta}^{\text {photon }}$ as the physical behavior of the system cannot depend on the choice of basis in $\mathcal{E}_{d}$. The simplest choice is an average of all channels, i.e.,

$$
\sigma_{\text {dressed }}^{\text {photon }}=\frac{1}{1+n_{d}} \sum_{\beta} \sigma_{\beta}^{\text {photon }},
$$

where $n_{d}$ is the number of degenerate states. Results for the case of $\sigma^{-}$polarization and a low intensity of $64 \mathrm{~mW} / \mathrm{cm}^{2}$ are shown in Fig. 2.

For the modified coupling method, the cross section $\sigma_{\alpha}^{\text {photon }}$ for the undressed channel $|\alpha\rangle$ is more straightforward to analyze in terms of experimental conditions. We choose to form a similar quantity

$$
\sigma_{\text {modified }}^{\text {photon }}=\frac{1}{n_{o}} \sum_{\alpha} \sigma_{\alpha}^{\text {photon }},
$$

where $n_{o}$ is the number of open channels and again fit the resonances to Fano profiles. In this way we can compare the profiles with those of the dressed-state calculation. Results for $\sigma^{-}$polarization and an intensity of $64 \mathrm{~mW} / \mathrm{cm}^{2}$ are shown in Fig. 3.

The two profiles shown in Figs. 2 and 3 are very similar apart from the suppression of the background at small detunings for the dressed-state profile. Importantly, the resonance parameters $E_{\text {res }}$ and $\Gamma_{\text {res }}$ obtained from fits to the two profiles are identical. The background loss behavior present in the profiles is due to the dominance of the off-diagonal Rabi couplings over the diagonal terms $\Delta E$ and $E_{K}$ of the potential matrix for the asymptotic region. This situation arises because of the shallow nature of the excited state potential and the ultracold temperature used. If the well is artificially deepened, or much larger kinetic energies are used, then this background completely disappears.

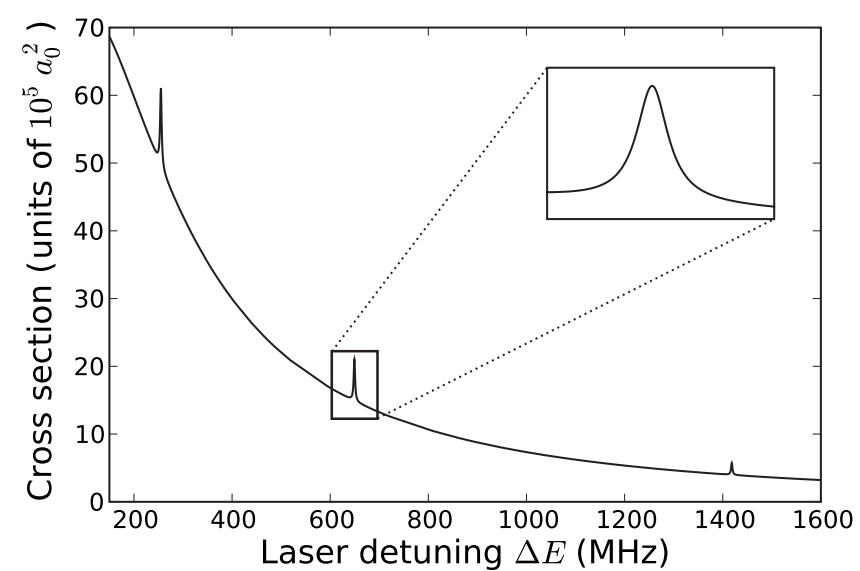

FIG. 3. PA cross-section profile calculated using the modified coupling. Results shown are for $\sigma^{-}$polarization and a low intensity of $64 \mathrm{~mW} / \mathrm{cm}^{2}$.

The PA profiles for high laser intensity show some unusual behavior. Results for the dressed-state and modified coupling profiles at an intensity of $2.6 \mathrm{~W} / \mathrm{cm}^{2}$ are shown in Figs. 4 and 5, respectively. These spectra exhibit two features not present in previously calculated PA profiles; strong interference between the resonance and background contributions and a reduction in the overall magnitude of the dressed profile. The interference feature is apparent in the magnitude of the corresponding Fano parameter $p=1 / q \sim 0.5$ indicating that these resonances exhibit a severe departure from Lorentzian-type behavior.

The dependence of the resonance position and width upon laser intensity is shown in Figs. 6 and 7, respectively, for the case of $\sigma^{-}$polarization. Similar behavior is obtained for $\sigma^{+}$ polarization. The intensity dependence of the line shift and width is very close to linear and quadratic, respectively, for $v=0,1$ but departures from these dependencies are evident for $v=2$. This can be seen from the fit parameters given in Table II which shows that the coefficients $s_{2}$ of the quadratic correction and $w_{3}$ of the cubic correction to the intensity dependence of the shift and width, respectively, for $v=0,1$ are very small.

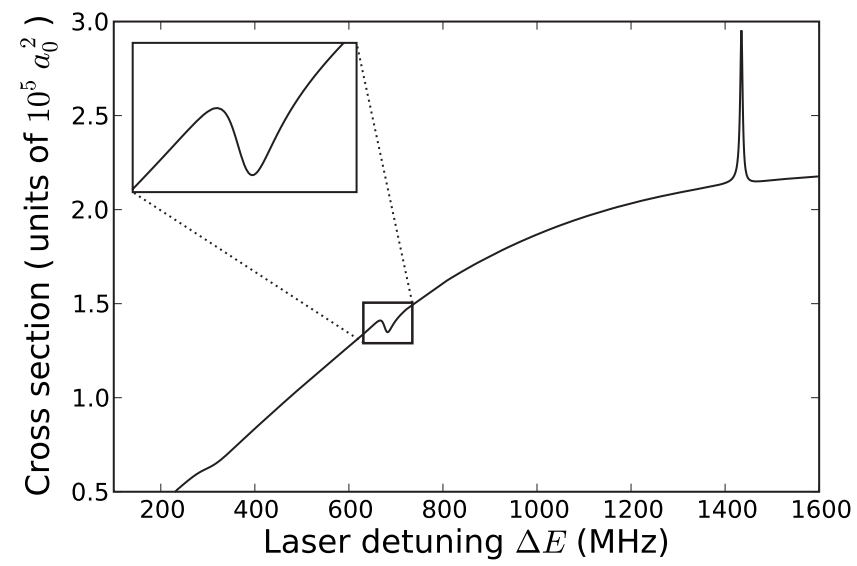

FIG. 4. PA cross-section profile calculated using dressed states. Results shown are for $\sigma^{-}$polarization and a high intensity of $2.6 \mathrm{~W} / \mathrm{cm}^{2}$. 


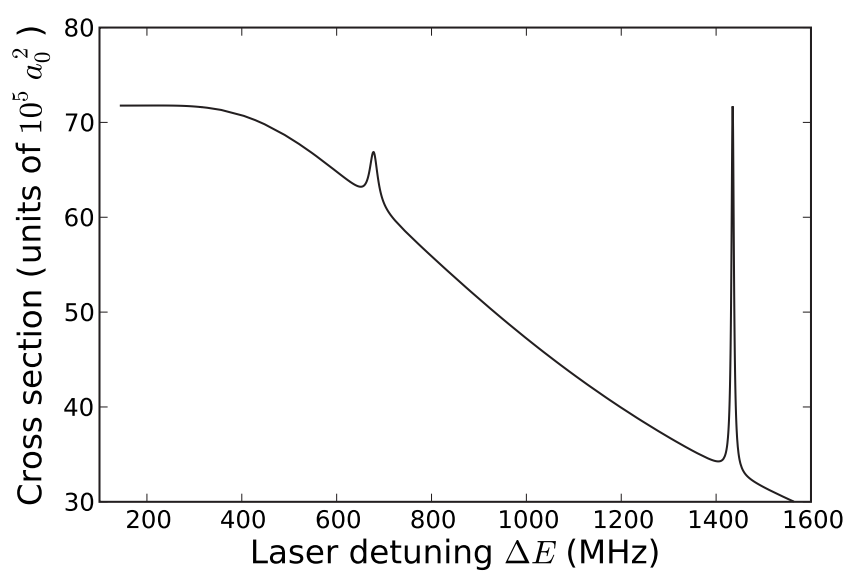

FIG. 5. PA cross-section profile calculated using the modified coupling. Results shown are for $\sigma^{-}$polarization and a high intensity of $2.6 \mathrm{~W} / \mathrm{cm}^{2}$.

\section{DISCUSSION AND CONCLUSIONS}

We have investigated the line shifts and widths for photoassociation of spin-polarized metastable helium to the three lowest rovibrational levels of the $J=1,0_{u}^{+}$state asymptoting to $2{ }^{3} S_{1}+2 p{ }^{3} P_{0}$ using two variants of a nonperturbative close-coupled treatment, one based upon dressed states and the other on a modified radiative coupling which vanishes asymptotically. We have also calculated the shifts using a second-order perturbative treatment.

The main physical interest is in the properties of the PA resonance profiles and our results for the shifts and widths of these profiles obtained using the two variants of the nonperturbative calculation agree to better than $2 \%$. Both methods indicate that there is significant background loss for this metastable helium system, a feature not present in studies of PA in other systems, which is due to the shallow nature of the excited state potential.

The behavior of the PA profiles for high laser intensity is quite different for the dressed-state and modified coupling methods. Physically, the behavior of the modified coupling

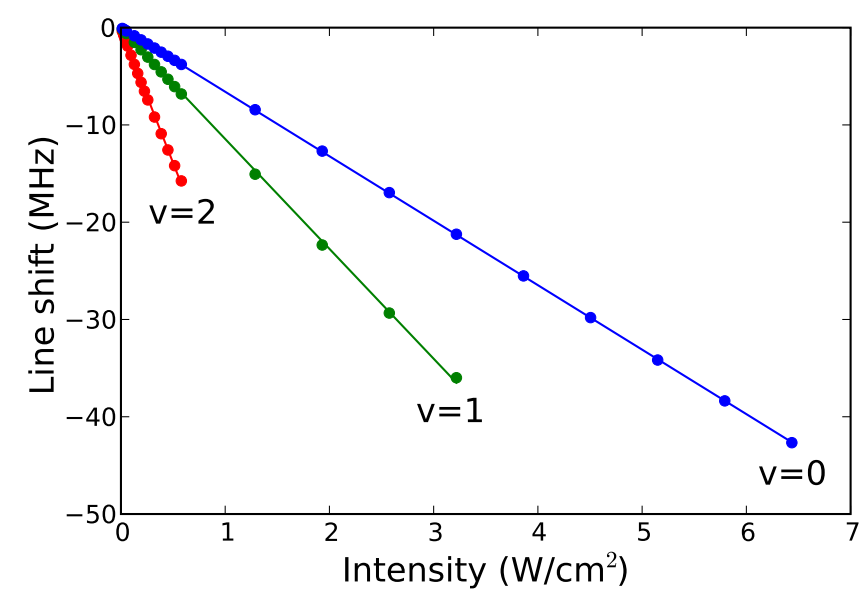

FIG. 6. (Color online) Dependence upon laser intensity of the line shift of the $v=0,1,2$ levels for $\sigma^{-}$polarization coupled to the $0_{u}^{+}$state.

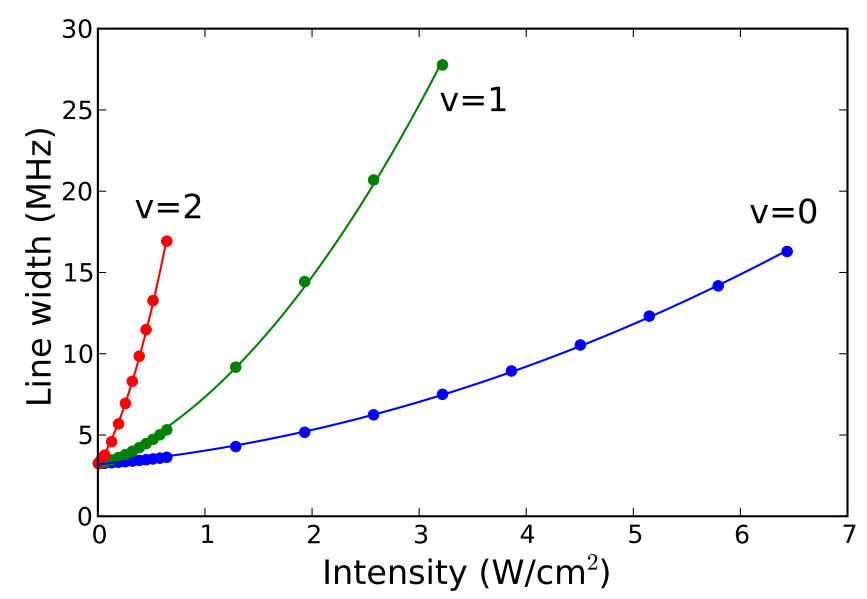

FIG. 7. (Color online) Dependence upon laser intensity of the linewidth of the $v=0,1,2$ levels for $\sigma^{-}$polarization coupled to the $0_{u}^{+}$state.

profile appears to make sense; if the laser is intense enough that most loss occurs outside the collision region then the particular resonance of the excited state will have little effect on the profile. Hence, a form of saturation is observed. Note that this first occurs in the higher vibrational levels as the intensity is increased. The behavior of the dressed-states profile is very different in that the overall magnitude of the profile decreases at higher intensities and lower detunings. It is difficult to relate this behavior to the collision process without the appropriate association of the dressed-state description with the experimental undressed states. When the laser is switched on, the initial undressed states must be transformed to the dressed states and in this process the undressed open channels acquire significant components of the pseudo-open and closed dressed channels and thereby suffer loss. In the limit of infinite intensity, the open dressed channels become completely uncoupled from the pseudo-open and closed channels, and the loss from the dressed states is entirely due to the activation of the laser. This suggests that the general interpretation of quantities constructed from dressed $S$-matrix elements at large intensities requires a proper description of the activation of the laser in the formalism. Fortunately, this detail is not required to obtain the resonance parameters as both treatments result in nearly identical shift and width values.

The results for the line shifts from the close-coupled and perturbative calculations agree very closely at low laser intensities. The $s_{1}$ values in Table II differ by $\lesssim 0.5 \%$ from our perturbative results (see Table I) calculated with the correction $V_{e}^{\mathrm{KC}}$ included. The small differences can be explained by the fact that a finite kinetic energy $(2.1 \mu \mathrm{K})$ was used in the close-coupled calculation whereas a zero kinetic energy was used in the perturbative calculation. We note that an increase in the kinetic energy by an order of magnitude decreases the nonperturbative line shift fit parameter by approximately $2 \%$.

At higher intensities the perturbative results remain a fair approximation to the nonperturbative results; however, small nonlinear differences are evident, affecting the line shifts by up to $5 \%$. Additionally, a maximum intensity is found that limits the visibility of the resonances. This laser saturation 
TABLE II. Parameters for a quadratic fit $s_{1} I+s_{2} I^{2}$ and cubic fit $w_{0}+w_{1} I+w_{2} I^{2}+w_{3} I^{3}$ to the dependence of the line shifts and the line full widths, respectively, (in $\mathrm{MHz}$ ) upon laser intensity $I\left(\mathrm{~W} / \mathrm{cm}^{2}\right)$. The value $I_{\max }$ denotes the approximate maximum intensity up to which the resonance peak is clearly discernable and there is no overlap with neighboring peaks.

\begin{tabular}{|c|c|c|c|c|c|c|c|c|}
\hline \multirow[b]{2}{*}{ Level } & \multirow[b]{2}{*}{ Polarization } & \multicolumn{2}{|c|}{ Shift } & \multicolumn{4}{|c|}{ Width } & \multirow[b]{2}{*}{$I_{\max }$} \\
\hline & & $s_{1}$ & $s_{2}$ & $w_{0}$ & $w_{1}$ & $w_{2}$ & $w_{3}$ & \\
\hline$v=0$ & $\sigma^{-}$ & -6.514 & -0.023 & 3.233 & 0.441 & 0.304 & -0.0093 & 7.0 \\
\hline$v=0$ & $\sigma^{+}$ & -7.781 & -0.021 & 3.226 & 0.730 & 0.329 & -0.0091 & 7.0 \\
\hline$v=1$ & $\sigma^{-}$ & -11.77 & 0.074 & 3.231 & 1.51 & 2.87 & -0.34 & 3.2 \\
\hline$v=1$ & $\sigma^{+}$ & -10.30 & -0.018 & 3.219 & 1.47 & 1.70 & -0.10 & 3.2 \\
\hline$v=2$ & $\sigma^{-}$ & -29.79 & 3.90 & 3.216 & 6.25 & 36.9 & -21.9 & 0.4 \\
\hline$v=2$ & $\sigma^{+}$ & -24.95 & 1.13 & 3.197 & 5.44 & 18.2 & -4.20 & 0.6 \\
\hline
\end{tabular}

should be observable in the laboratory and suggests that ultracold photoassociation to shallow potentials is only useful at lower laser intensities. Although not reported here, it can be shown [5] that the perturbative linewidth is also linearly dependent upon laser intensity. This is obviously not true in the present nonperturbative calculation, where a significant quadratic behavior is observed.

We conclude by noting that our theoretical findings are consistent with the limited experimental data available [17]. Our values for the shift ratios $s_{1}(v=1) / s_{1}(v=0)$ and $s_{1}(v$ $=2) / s_{1}(v=0)$ of 1.81 and 4.57 for $\sigma^{-}$polarization lie within the experimental values of $1.71 \pm 0.14$ and $4.20 \pm 0.48$, respectively, and our $v=0$ widths of $3.24 \mathrm{MHz}$ at $9 \mathrm{~mW} / \mathrm{cm}^{2}$ and $11.9 \mathrm{MHz}$ at $5 \mathrm{~W} / \mathrm{cm}^{2}$ are comparable to the experimental values estimated from Fig. 2 of [17] of $3 \mathrm{MHz}$ and 10 $\mathrm{MHz}$, respectively.

\section{ACKNOWLEDGMENT}

I.B.W. would like to thank A. S. Dickinson for helpful discussions and the suggestion that we investigate the use of a modified radiative coupling.

\section{APPENDIX A: THE $\left|j_{1} j_{2} j \Omega_{j}, J m_{J} p\right\rangle$ BASIS}

In the $j j$ coupling scheme, the total angular momenta $\boldsymbol{j}_{i}$ of each atom are coupled to form the total electronic angular momentum $\boldsymbol{j}=\boldsymbol{j}_{1}+\boldsymbol{j}_{2}$ which is then coupled to the relative angular momentum $l$ of the nuclei to form the total angular momentum $\boldsymbol{J}=\boldsymbol{j}+\boldsymbol{l}$. This gives the basis states in the spacefixed reference frame

$$
\left|\gamma j_{1} j_{2} j l J m_{J}\right\rangle=\sum_{m_{j} m_{l}} C_{m_{j} m_{l} m_{J}}^{j l J}\left|\gamma j_{1} j_{2} j m_{j}\right\rangle\left|l m_{l}\right\rangle,
$$

where $\left|\gamma j_{1} j_{2} j m_{j}\right\rangle$ are the eigenstates of the two asymptotically free atoms and $\left|l m_{l}\right\rangle=Y_{l, m_{l}}(\theta, \phi)$ are the relative motion eigenstates. Here $\gamma=\left(\gamma_{1}, \gamma_{2}\right)$ and $\gamma_{i}=\left\{\bar{\gamma}_{i}, L_{i}, S_{i}\right\}$, where $\boldsymbol{L}_{i}$ and $S_{i}$ are the total orbital and total spin angular momentum, respectively, of the individual atoms. The label $\bar{\gamma}_{i}$ denotes any additional quantum numbers needed including those specifying the electron configurations. All subscripted $m$ quantities denote projections on the $O z$ axis of the spacefixed frame.

The transformation from the space-fixed frame to the molecular frame is

$$
\left|\gamma j_{1} j_{2} j m_{j}\right\rangle=\sum_{\Omega_{j}} D_{m_{j} \Omega_{j}}^{j *}(\phi, \theta, 0)\left|\gamma j_{1} j_{2} j \Omega_{j}\right\rangle,
$$

where subscripted $\Omega$ quantities indicate projections along the intermolecular axis and $D_{m_{j} \Omega_{j}}^{j}$ is the Wigner rotation matrix [38]. Expressing the relative motion state $\left|m_{l}\right\rangle$ as a rotation matrix gives

$$
\begin{aligned}
\left|\gamma j_{1} j_{2} j l J m_{J}\right\rangle= & \sum_{m_{j} m_{l} \Omega_{j}} C_{m_{j} m_{l} m_{J}}^{j l J} \sqrt{\frac{2 l+1}{4 \pi}} D_{m_{l} 0}^{l *}(\phi, \theta, 0) D_{m_{j} \Omega_{j}}^{j *} \\
& \times(\phi, \theta, 0)\left|\gamma j_{1} j_{2} j \Omega_{j}\right\rangle .
\end{aligned}
$$

Combining the rotation matrices and using the sum rule for Clebsch-Gordan coefficients reduces Eq. (A3) to

$$
\left|\gamma j_{1} j_{2} j l J m_{J}\right\rangle=\sum_{\Omega_{j}}(-1)^{j-\Omega_{j}} C_{\Omega_{j}-\Omega_{j} 0}^{j J l} N_{m_{J} \Omega_{j}}^{J}(\theta, \phi)\left|\gamma j_{1} j_{2} j \Omega_{j}\right\rangle,
$$

where $N_{m_{J} \Omega_{j}}^{J}$ is the symmetric top function defined as

$$
N_{m_{J} \Omega_{J}}^{J} \equiv \sqrt{\frac{2 J+1}{4 \pi}} D_{m_{J} \Omega_{J}}^{J *}(\phi, \theta, 0) .
$$

Equation (A4) can be interpreted as a coupling of $j$ and $J$ to result in $l$ and naturally introduces the basis states

$$
\left|\gamma j_{1} j_{2} j \Omega_{j} J m_{J}\right\rangle \equiv N_{m_{J} \Omega_{j}}^{J}\left|\gamma j_{1} j_{2} j \Omega_{j}\right\rangle .
$$

The derivation of Eq. (A6) has not taken into account any of the symmetry requirements of the system. Following [39], we define symmetric states that are eigenstates of the operator $\hat{I}$, the inversion operator of the total wave function through the center of charge of the molecule. The eigenvalues of $\hat{I}$ are $(-1)^{w}$ with $w=0$ for gerade symmetry and $w$ $=1$ for ungerade symmetry. For $\Omega_{j}=0$ states, we can also identify the quantum number of $\hat{\sigma}_{v}$, the reflection operator of the total wave function through a plane containing the inter- 
molecular axis. For identical nuclei the symmetric states for $j j$ and $L S$ couplings are

$$
\left|\gamma_{1} j_{1} \gamma_{2} j_{2} j \Omega_{j} w\right\rangle=N_{j j}\left[\left|\gamma_{1} j_{1} \gamma_{2} j_{2} j \Omega_{j}\right\rangle+(-1)^{p_{j j}}\left|\gamma_{2} j_{2} \gamma_{1} j_{1} j \Omega_{j}\right\rangle\right]
$$

and

$$
\begin{aligned}
\left|\gamma_{1} \gamma_{2} L S \Omega_{L} \Omega_{S} w\right\rangle= & N_{L S}\left[\left|\gamma_{1} \gamma_{2} L S \Omega_{L} \Omega_{S}\right\rangle\right. \\
& \left.+(-1)^{p_{L S}}\left|\gamma_{2} \gamma_{1} L S \Omega_{L} \Omega_{S}\right\rangle\right]
\end{aligned}
$$

where the explicit ordering of $\gamma_{1}$ and $\gamma_{2}$ indicates the order in which the angular momenta are coupled and $p$, which labels the symmetry under permutation of the labels $1 \leftrightarrow 2$, is uniquely related to $w$. For the $j j$ state (A7), $p_{j j}=w+w_{1}+w_{2}$ $+N+j_{1}+j_{2}-j$, where $w_{i}$ is the symmetry under inversion of the electronic wave function of atom $i$ about its nucleus and $N$ is the total number of electrons per atom. The $L S$ state (A8) has $p_{L S}=w+w_{1}+w_{2}+N+S_{1}+S_{2}-S+L_{1}+L_{2}-L$.

The normalization constants $N_{j j}$ and $N_{L S}$ are

$$
N_{j j}=\left\{\begin{array}{cc}
\frac{1}{\sqrt{2}} & \text { for }\left(\gamma_{1}, j_{1}\right) \neq\left(\gamma_{2}, j_{2}\right) \\
\frac{1}{2} & \text { for }\left(\gamma_{1}, j_{1}\right)=\left(\gamma_{2}, j_{2}\right)
\end{array}\right.
$$

and

$$
N_{L S}= \begin{cases}\frac{1}{\sqrt{2}} & \text { for } \gamma_{1} \neq \gamma_{2} \\ \frac{1}{2} & \text { for } \gamma_{1}=\gamma_{2}\end{cases}
$$
is

The transformation between the two bases (A7) and (A8)

$$
\left|\gamma_{1} j_{1} \gamma_{2} j_{2} j \Omega_{j} w\right\rangle=\sum_{L S \Omega_{L} \Omega_{S}} \frac{N_{j j}}{N_{L S}} F_{L S \Omega_{L} \Omega_{S}}^{j_{1} j_{2} \Omega_{j}}\left|\gamma_{1} \gamma_{2} L S \Omega_{L} \Omega_{S} w\right\rangle
$$

where

$$
\begin{aligned}
F_{L S \Omega_{L} \Omega_{S}}^{j_{1} j_{2} j \Omega_{j}}= & {\left[(2 S+1)(2 L+1)\left(2 j_{1}+1\right)\right.} \\
& \left.\times\left(2 j_{2}+1\right)\right]^{1 / 2} C_{m_{L} m_{S} m_{j}}^{L S j}\left\{\begin{array}{ccc}
L_{1} & L_{2} & L \\
S_{1} & S_{2} & S \\
j_{1} & j_{2} & j
\end{array}\right\},
\end{aligned}
$$

where the $\{\cdots\}$ is the Wigner $9-j$ symbol and the implicit set of quantum numbers $\left(\gamma_{1}, \gamma_{2}\right)$ has been suppressed.

\section{APPENDIX B: MATRIX ELEMENTS}

\section{Kinetic terms}

The radial kinetic term has the form

$$
\begin{aligned}
\left\langle a^{\prime}\left|\hat{T} \frac{1}{R} G_{a}(R)\right| a\right\rangle= & -\frac{\hbar^{2}}{2 \mu R}\left(\frac{d^{2} G_{a}}{d R^{2}} \delta_{a a^{\prime}}+2 \frac{d G_{a}}{d R}\left\langle a^{\prime}\right| \frac{d|a\rangle}{d R}\right. \\
& \left.+G_{a}\left\langle a^{\prime}\right| \frac{d^{2}|a\rangle}{d R^{2}}\right)
\end{aligned}
$$

where $|a\rangle \equiv\left|\Phi_{a}(R, q)\right\rangle$ and $a$ represents the quantum numbers $\left\{\gamma_{1}, \gamma_{2}, j_{1}, j_{2}, j, \Omega_{j}, w, J, m_{J}\right\}$. As the basis states are assumed to vary little with respect to $R$, the last two terms in Eq. (B1) are negligible at the long ranges considered in this investigation.

In this investigation the adiabatic excited state $\left|\psi_{e}^{0}\right\rangle$ is a combination of these basis states [see Eq. (13)] and the coefficients $C_{e a}(R)$ do vary considerably with $R$. If we assume no other excited states are coupled to the system (i.e., the adiabatic approximation is exact) then we only require the radial term (B1) with $|a\rangle=\left|a^{\prime}\right\rangle=\left|\psi_{e}^{0}\right\rangle$. The second term of Eq. (B1) is then zero as

$$
\left\langle\left.\psi_{e}^{0}\left|\frac{d\left|\psi_{e}^{0}\right\rangle}{d R}=\frac{1}{2} \frac{d}{d R} \sum_{a}\right| C_{e a}(R)\right|^{2}=0 .\right.
$$

The third term, however, is nonzero and gives rise to the kinetic correction term

$$
V_{e}^{\mathrm{KC}}=-\frac{\hbar^{2}}{2 \mu R} \sum_{a} C_{e a}(R) \frac{d^{2} C_{e a}(R)}{d R^{2}} .
$$

The matrix elements of the rotational kinetic term

$$
\hat{H}_{\text {rot }}=\frac{\hat{l}^{2}}{2 \mu R^{2}}
$$

are evaluated using the expansion of $\hat{l}^{2}$ in terms of ladder operators

$$
\hat{l}^{2}=\hat{J}^{2}+\hat{j}^{2}-\left(2 \hat{J}_{z} \hat{j}_{z}+\hat{J}_{+} \hat{j}_{-}+\hat{J}_{-} \hat{j}_{+}\right)
$$

where the subscripts refer to molecule-fixed axes, $\hat{J}_{ \pm}$ $\equiv \hat{J}_{x} \pm i \hat{J}_{y}$, and $\hat{j}_{ \pm} \equiv \hat{j}_{x} \pm i \hat{j}_{y}$. The action of $\hat{J}_{ \pm}$is irregular [40] due to the rotation of $N_{m_{J} \Omega_{j}}^{J}$ and is given by

$$
\hat{J}_{ \pm} N_{m_{J} \Omega_{j}}^{J}=\hbar \sqrt{J(J+1)-\Omega_{j}\left(\Omega_{j} \mp 1\right)} N_{m_{J} \Omega_{j} \mp 1}^{J} .
$$

Hence, the matrix elements of $\hat{l}^{2}$ are

$$
\begin{aligned}
\left\langle a^{\prime}\left|l^{2}\right| a\right\rangle= & \hbar^{2} \delta_{\rho \rho^{\prime}}\left\{\left[J(J+1)+j(j+1)-2 \Omega_{j}^{2}\right] \delta_{\Omega_{j}^{\prime} \Omega_{j}}\right. \\
& \left.-K_{J j \Omega_{j}}^{-} \delta_{\Omega_{j}^{\prime}, \Omega_{j}-1}-K_{J j \Omega_{j}}^{+} \delta_{\Omega_{j}^{\prime}, \Omega_{j}+1}\right\},
\end{aligned}
$$

where $|a\rangle \equiv\left|\Phi_{a}(R, q)\right\rangle$, a represents the quantum numbers $\left\{\gamma_{1}, \gamma_{2}, j_{1}, j_{2}, j, \Omega_{j}, w, J, m_{J}\right\}, \rho$ denotes the set of quantum numbers $\left\{\gamma_{1}, \gamma_{2}, j_{1}, j_{2}, j, w, J, m_{J}\right\}$, and

$$
K_{J j \Omega_{j}}^{ \pm}=\left[J(J+1)-\Omega_{j}\left(\Omega_{j} \pm 1\right)\right]^{1 / 2}\left[j(j+1)-\Omega_{j}\left(\Omega_{j} \pm 1\right)\right]^{1 / 2} .
$$

The terms nondiagonal in $\Omega_{j}$ are called the Coriolis couplings and are often negligible. This is the case for purely long-range bound states in metastable helium [14]. 


\section{Electronic term}

We wish to express the matrix elements of $\hat{H}_{\mathrm{el}}$ in terms of the Born-Oppenheimer potentials ${ }^{2 S+1} \Lambda_{w}^{ \pm}(R)$ defined by the eigenvalue equation

$$
\hat{H}_{\mathrm{el}}\left|L S \Omega_{L} \Omega_{S} w\right\rangle=\left[{ }^{2 S+1} \Lambda_{w}^{ \pm}(R)+E^{\infty}\right]\left|L S \Omega_{L} \Omega_{S} w\right\rangle,
$$

where $\Lambda \equiv\left|\Omega_{L}\right|$ and $E^{\infty}$ is the asymptotic energy of the state.

The matrix elements in basis (A7) are evaluated by transforming to basis (A8) using Eq. (A11) and then applying Eq. (B9) to obtain

$$
\begin{aligned}
\left\langle a^{\prime}\left|\hat{H}_{\mathrm{el}}\right| a\right\rangle= & \delta_{\eta \eta^{\prime}} \sum_{L S \Omega_{L} \Omega_{S}} \frac{N_{j j}^{2}}{N_{L S}^{2}} F^{j_{1}^{\prime} j_{2}^{\prime} j^{\prime} \Omega_{j}} \\
& \times\left[S \Omega_{L} \Omega_{S}\right. \\
& \times\left[{ }^{2 S+1} \Lambda_{w}^{ \pm}(R)+E_{a}^{\infty}\right] F_{L S \Omega_{L} \Omega_{S}}^{j_{1} j_{2} j \Omega_{j}},
\end{aligned}
$$

where $\eta=\left\{\gamma_{1}, \gamma_{2}, \Omega_{j}, w, J, m_{J}\right\}$. For our case of metastable helium involving the $2 s 2 s$ and $2 s 2 p$ states, $N_{j j}=N_{L S}$.

\section{Fine-structure term}

The total fine-structure term $\hat{H}_{\mathrm{fs}}$ is the sum of the finestructure terms for the individual atoms and is diagonal in basis (A6) in the asymptotic region of free atoms. For the long-range molecular states considered in this work, the total atomic angular momenta are considered to be approximately good quantum numbers. Hence, the matrix elements of the total fine-structure term are

$$
\left\langle a^{\prime}\left|\hat{H}_{\mathrm{fs}}\right| a\right\rangle=\left\langle a^{\prime}\left|\hat{H}_{\mathrm{fs}}^{1}+\hat{H}_{\mathrm{fs}}^{2}\right| a\right\rangle=\delta_{a a^{\prime}}\left(\Delta E_{\gamma_{1} j_{1}}^{\mathrm{fs}}+\Delta E_{\gamma_{2} j_{2}}^{\mathrm{fs}}\right),
$$

where $\hat{H}_{\mathrm{fs}}^{i}$ represents the fine-structure interaction and $\Delta E_{\gamma_{i} j_{i}}^{\mathrm{fs}}$ the fine-structure splittings for atom $i$.

\section{Laser interaction term}

For radiation of a given circular polarization $\boldsymbol{\epsilon}_{\lambda}$ in the space-fixed frame, where $\lambda=0, \pm 1$ for $\pi, \sigma^{ \pm}$polarization, laser-matter interaction (10) can be expanded in a spherical basis using

$$
\boldsymbol{\epsilon}_{\lambda} \cdot \boldsymbol{d}=\sum_{\xi=0, \pm 1}(-1)^{\xi}\left(\boldsymbol{\epsilon}_{\lambda}\right)_{-\xi} d_{\xi}
$$

where $\left(\boldsymbol{\epsilon}_{\lambda}\right)_{-\xi}=\delta_{\lambda, \xi}$. The matrix elements of $\hat{H}_{\text {int }}$ are, after rotation to the molecular frame and transformation to the $L S$ basis states,

$$
\begin{aligned}
\left\langle a^{\prime}\left|\hat{H}_{\mathrm{int}}\right| a\right\rangle= & A_{\lambda} \frac{N_{j j}^{\prime}}{N_{L S}^{\prime}} \frac{N_{j j}}{N_{L S}} \sum_{L^{\prime} S^{\prime} \Omega_{L^{\prime} \Omega_{S}^{\prime}}} \sum_{L S \Omega_{L} \Omega_{S}} F_{L^{\prime} S^{\prime} \Omega_{L}^{\prime} \Omega_{S}^{\prime}}^{j_{1}^{\prime} j_{2}^{\prime} j^{\prime} \Omega_{j}^{\prime}} F_{L S \Omega_{L} \Omega_{S} j_{2} j \Omega_{j}} \\
& \times \int \sin \theta d \theta d \phi N_{m_{J^{\prime}} \Omega_{j}^{\prime}}^{J^{\prime}} D_{\lambda b}^{1 *} N_{m_{J} \Omega_{j}}^{J} \\
& \times\left\langle\gamma^{\prime} L^{\prime} S^{\prime} \Omega_{L^{\prime}}^{\prime} \Omega_{S}^{\prime} w^{\prime}\left|d_{b}\right| \gamma L S \Omega_{L} \Omega_{S} w\right\rangle,
\end{aligned}
$$

where $A_{\lambda}=(-1)^{\lambda} \sqrt{I / 2 \epsilon_{0} c}, D_{m_{j} \Omega_{j}}^{j} \equiv D_{m_{j} \Omega_{j}}^{j}(\theta, \phi, 0)$, and $b$ labels the spherical basis components in the molecular frame.

The terms involving $J$ and $J^{\prime}$ can be expanded and the integration over the interatomic polar coordinates performed to give

$$
\begin{aligned}
& \sqrt{\frac{\left(2 J^{\prime}+1\right)(2 J+1)}{4 \pi}} \int \sin \theta d \theta d \phi D_{m_{J}^{\prime} \Omega_{j}^{\prime}}^{J^{\prime}} D_{\lambda b}^{1 *} D_{m_{J} \Omega_{j}}^{J *} \\
& =\sqrt{\frac{2 J+1}{2 J^{\prime}+1}} C_{m_{J^{\prime} m_{J}^{\prime}}}^{J J^{\prime}} C_{\Omega_{j} b \Omega_{j}^{\prime}}^{J 1 J^{\prime}} .
\end{aligned}
$$

The matrix element of $d_{b}=d_{b}^{1}+d_{b}^{2}$ between $L S$ states must be evaluated under proper symmetry considerations [26]. For the helium $2 s-2 p\left(0_{u}^{+}\right)$transition this results in

$$
\begin{aligned}
& \left\langle L^{\prime}(=1) S^{\prime} \Omega_{L}^{\prime} \Omega_{S}^{\prime} w^{\prime}(=1)\left|d_{b}\right| L(=0) S \Omega_{L}(=0) \Omega_{S} w\right\rangle \\
& \quad=\delta_{S S^{\prime}} \delta_{\Omega_{S} \Omega_{S}^{\prime}} \delta_{b \Omega_{L}^{\prime}} \frac{d_{\mathrm{at}}^{\mathrm{sp}}}{\sqrt{2}}\left[1+(-1)^{1+S+w^{\prime}}\right]
\end{aligned}
$$

where $d_{\mathrm{at}}^{\mathrm{sp}}$ is the reduced matrix element of the dipole operator between the $2 s$ and $2 p$ atomic states. Only gerade ( $w$ $=0)$ ground states are coupled to the excited state, and because metastable states must satisfy $(-1)^{S+w}=1$ due to their bosonic nature, only ${ }^{1} \Sigma_{g}^{+}$and ${ }^{5} \Sigma_{g}^{+}$states are coupled to the excited state.

After simplification the complete matrix element becomes

$$
\begin{aligned}
\left\langle a^{\prime}\left|\hat{H}_{\mathrm{int}}\right| a\right\rangle= & (-1)^{\lambda} \sqrt{\frac{I}{\epsilon_{0} c}} \sqrt{\frac{2 J+1}{2 J^{\prime}+1}} F_{1, j,-\Omega_{j}, \Omega_{j}}^{j_{1}^{\prime} j_{j}^{\prime} j^{\prime} 0} \\
& \times C_{\Omega_{j},-\Omega_{j}, 0}^{J 1 J^{\prime}} C_{m_{J}, \lambda, m_{J}^{\prime}}^{J 1 J^{\prime}} d_{\mathrm{at}}^{\mathrm{sp}}
\end{aligned}
$$

assuming that $w^{\prime}=1$ and $w=0$.
[1] H. R. Thorsheim, J. Weiner, and P. S. Julienne, Phys. Rev. Lett. 58, 2420 (1987).

[2] J. Weiner, V. S. Bagnato, S. Zilio, and P. S. Julienne, Rev. Mod. Phys. 71, 1 (1999).

[3] C. McKenzie et al., Phys. Rev. Lett. 88, 120403 (2002); C. Samuelis, S. Falke, T. Laue, P. Pellegrini, O. Dulieu, H. Knockel, and E. Tiemann, Eur. Phys. J. D 26, 307 (2003).

[4] J. M. Gerton, B. J. Frew, and R. G. Hulet Phys. Rev. A 64,
053410 (2001); I. D. Prodan, M. Pichler, M. Junker, R. G. Hulet, and J. L. Bohn, Phys. Rev. Lett. 91, 080402 (2003).

[5] M. Portier, S. Moal, J. Kim, M. Leduc, C. Cohen-Tannoudji, and O. Dulieu, J. Phys. B 39, S881 (2006).

[6] R. G. Dall, K. G. H. Baldwin, L. J. Byron, and A. G. Truscott, Phys. Rev. Lett. 100, 023001 (2008); N. Bouloufa, A. Crubellier, and O. Dulieu, Phys. Scr. T134, 014014 (2009).

[7] P. S. Julienne, J. Res. Natl. Inst. Stand. Technol. 101, 487 
(1996).

[8] A. Fioretti, D. Comparat, A. Crubellier, O. Dulieu, F. MasnouSeeuws, and P. Pillet, Phys. Rev. Lett. 80, 4402 (1998); K. M. Jones, E. Tiesinga, P. D. Lett, and P. S. Julienne, Rev. Mod. Phys. 78, 483 (2006); P. S. Julienne, Faraday Discuss. 142, 361 (2009).

[9] N. Herschbach, P. J. J. Tol, W. Vassen, W. Hogervorst, G. Woestenenk, J. W. Thomsen, P. van der Straten, and A. Niehaus, Phys. Rev. Lett. 84, 1874 (2000).

[10] J. Léonard, M. Walhout, A. P. Mosk, T. Müller, M. Leduc, and C. Cohen-Tannoudji, Phys. Rev. Lett. 91, 073203 (2003).

[11] J. Kim, U. D. Rapol, S. Moal, J. Léonard, M. Walhout, and M. Leduc, Eur. Phys. J. D 31, 227 (2004).

[12] M. van Rijnbach, Ph.D. thesis, University of Utrecht, 2004.

[13] J. Léonard, A. P. Mosk, M. Walhout, P. van der Straten, M. Leduc, and C. Cohen-Tannoudji, Phys. Rev. A 69, 032702 (2004).

[14] V. Venturi, P. J. Leo, E. Tiesinga, C. J. Williams, and I. B. Whittingham, Phys. Rev. A 68, 022706 (2003).

[15] A. S. Dickinson, F. X. Gadéa, and T. Leininger, Europhys. Lett. 70, 320 (2005).

[16] B. Deguilhem, T. Leininger, F. X. Gadéa, and A. S. Dickinson, J. Phys. B 42, 015102 (2009).

[17] J. Kim, S. Moal, M. Portier, J. Degué, M. Leduc, and C. Cohen-Tannoudji, Europhys. Lett. 72, 548 (2005).

[18] S. Moal, M. Portier, J. Kim, J. Dugué, U. D. Rapol, M. Leduc, and C. Cohen-Tannoudji, Phys. Rev. Lett. 96, 023203 (2006).

[19] M. Przybytek and B. Jeziorski, J. Chem. Phys. 123, 134315 (2005).

[20] R. Napolitano, J. Weiner, C. J. Williams, and P. S. Julienne, Phys. Rev. Lett. 73, 1352 (1994).

[21] J. L. Bohn and P. S. Julienne, Phys. Rev. A 60, 414 (1999).
[22] A. Simoni, P. S. Julienne, E. Tiesinga, and C. J. Williams, Phys. Rev. A 66, 063406 (2002).

[23] R. Napolitano, Phys. Rev. A 57, 1164 (1998).

[24] M. Leduc (private communication).

[25] F. H. Mies, Theoretical Chemistry: Advances and Perspectives, edited by D. Henderson (Academic, New York, 1981), pp. 127-198.

[26] J. Burke, Ph.D. thesis, University of Colorado, 1999.

[27] A. Dalgarno and J. T. Lewis, Proc. R. Soc. London, Ser. A 233, 70 (1955).

[28] E. Merzbacher, Quantum Mechanics, 3rd ed. (John Wiley and Sons, New York, 1998), Chap. 18.

[29] T. J. Beams, G. Peach, and I. B. Whittingham, J. Phys. B 37, 4561 (2004).

[30] T. J. Beams, G. Peach, and I. B. Whittingham, Phys. Rev. A 74, 014702 (2006).

[31] J. C. Light and T. Carrington, Adv. Chem. Phys. 114, 263 (2000).

[32] M. W. Müller, A. Merz, M.-W. Ruf, H. Hotop, W. Meyer, and M. Movre, Z. Phys. D: At., Mol. Clusters 21, 89 (1991).

[33] W. J. Meath, J. Chem. Phys. 48, 227 (1968).

[34] M. Marinescu (private communication).

[35] F. H. Mies, Mol. Phys. 41, 953 (1980).

[36] U. Fano, Phys. Rev. 124, 1866 (1961); U. Fano and J. W. Cooper, ibid. 137, A1364 (1965).

[37] B. R. Johnson, J. Chem. Phys. 69, 4678 (1978).

[38] D. M. Brink and G. R. Satchler, Angular Momentum, 2nd ed. (Clarendon Press, Oxford, 1968).

[39] E. E. Nikitin and S. Ya. Umanskii, Theory of Slow Atomic Collisions, 1st ed. (Springer, Berlin, 1984).

[40] J. H. van Vleck, Rev. Mod. Phys. 23, 213 (1951). 